\title{
Molecular dynamics studies of polar/nonpolar fluid mixtures. I. Mixtures of Lennard-Jones and Stockmayer fluids
}

\author{
S. W. de Leeuw \\ Laboratory for Physical Chemistry, University of Amsterdam, Nieuwe Achtergracht 127, 1018 WS \\ Amsterdam, The Netherlands \\ B. Smit and C. P. Williams \\ Koninklijke/Shell-Laboratorium, Amsterdam, (Shell Research B. V.), P.O. Box 3003, 1003 AA \\ Amsterdam, The Netherlands
}

(Received 23 January 1990; accepted 4 May 1990)

\begin{abstract}
The results of canonical ensemble molecular dynamics calculations of mixtures of LennardJones and Stockmayer fluids are reported. To study solely the influence of the polarity, the Lennard-Jones parameters were identical for both components. The excess mixing properties show a strong asymmetry with respect to composition for large dipolar strength. The free energy of mixing is obtained through a thermodynamic integration procedure. The results strongly suggest that, for reduced dipolar strengths $\mu^{2}>3.15$, demixing occurs into a phase rich in polar component and an almost pure Lennard-Jones fluid. It is shown that perturbation theory yields fairly accurate results for the dipolar energy and free energy of the mixture. For the free energy of mixing, qualitatively correct results are obtained. The structure and orientational correlation functions of the mixture are discussed. The radial distribution function for pairs of polar molecules show a marked increase in local ordering with dipolar strength for low concentrations of the polar component, indicating that strong clustering of polar molecules occurs at these concentrations. The orientational order is also seen to increase very strongly with dipole moment at these concentrations. The pair correlation function for pairs of Lennard-Jones atoms shows little dependence on dipolar strength of Stockmayer molecules at these concentrations. The distribution function for pairs of unlike molecules reflects the increasingly dissimilar character of these molecules as the dipolar strength increases. For large concentrations of Stockmayer molecules, the opposite effect is observed, albeit less pronounced, in that the pair-correlation function for Lennard-Jones atoms shows an increase in local ordering as $\mu$ increases, whereas the radial distribution function for Stockmayer pairs remains relatively unaffected with increasing $\mu$. These results are interpreted in terms of a frustation model. Results are given for the variation of the dielectric constant of the mixture with composition and dipolar strength.
\end{abstract}

\section{INTRODUCTION}

Polar/nonpolar fluid mixtures form a technologically important class of systems, whose thermodynamic and phase behavior is at present poorly understood and predicted. Mixtures of fluids consisting of polar molecules having spherical cores provide a convenient model to study the effects of polarity on the microscopic structure and thermodynamic behavior of these systems. The mixing behavior of these systems has been studied with thermodynamic perturbation theory by Gubbins and Twu. ${ }^{1,2}$ They extended the approach based on a Padé approximant, proposed by Stell, Rasaiah, and Narang, ${ }^{3,4}$ for the free energy expansion to mixtures of polar fluids. Using this approach, Gubbins et al. investigated the phase behavior of these mixtures for a wide range of potential parameters and showed that these simple models exhibited a rich diversity in phase behavior. ${ }^{5}$ More recently, Morriss and Isbister ${ }^{6}$ used an integral equation approach to study mixtures of polar and nonpolar hard dumbells and predicted a phase separation into an almost pure nonpolar component and a mixture rich in polar component.

Surprisingly few computer simulations have been reported for such mixtures. Thus, at present it is not known whether perturbation theory gives a good description of the thermodynamic properties of such mixtures. A major problem in these simulations is the accurate determination of the phase boundaries in systems in which demixing occurs.

In this paper, we describe the results of molecular dynamics simulations of mixtures of Stockmayer and Lennard-Jones fluids of equal size. We investigate the variation of thermodynamic properties (pressure, energy, and excess mixing properties) with dipole moment. The excess energy of mixing is positive and exhibits the characteristic asymmetry, with a maximum at concentrations rich in nonpolar component, so often observed experimentally in mixtures of polar and nonpolar fluids. ${ }^{7}$

Through a thermodynamic integration procedure, we compute the free energy of mixing directly from the dipolar energy obtained from our molecular dynamics simulations. Our results show clearly that phase separation occurs in these systems for sufficiently large dipole moments of the polar component, in qualitative agreement with thermodynamic perturbation theory. We find, however, that the onset of demixing occurs for higher values of the dipolar strength $\mu^{2}$ than predicted by perturbation theory. Also, the compositions of the fluid phases in equilibrium differ from those pre- 
dicted by perturbation theory.

We have also computed the center of mass (COM) distribution functions and the leading spherical harmonic coefficients in the full pair correlation functions. These also show a marked asymmetry in their behavior with respect to composition. As the concentration of the polar component decreases, a strong increase in the orientational ordering for the polar molecules is observed. Also, the main peak in the COM distribution function for polar molecules attains a sharp first maximum at these concentrations, reflecting the onset of phase separation. Such a behavior has also been observed in Monte Carlo calculations of mixtures of quadrupolar molecules with spherical cores. ${ }^{5}$ The results can be explained qualitatively on the basis of a frustration model. At low concentrations of the polar component, mainly pairs of dipoles can be found close to each other so that the dipoles can orient themselves freely in energetically favorable (head-to-tail) configurations. As the concentration of the polar component increases, more contacts between dipoles will be unavoidable and the orientation of the dipoles will be the result of several competing pair orientations. The observed asymmetry leads to the asymmetry in the excess properties of mixing with respect to composition.

\section{COMPUTATIONAL DETAILS}

The systems we study consist of $N_{S}$ Stockmayer molecules and $N_{\mathbf{L J}}$ Lennard-Jones atoms. The potential energy of these systems is given by

$$
\begin{aligned}
V\left(\mathbf{R}_{1}, \ldots, \mathbf{R}_{N}, \boldsymbol{\mu}_{1}, \ldots, \boldsymbol{\mu}_{N}\right) \\
=\frac{1}{2} \sum_{i=1}^{N} \sum_{j \neq i}^{N} \phi_{\mathrm{LJ}}\left(\left|\mathbf{R}_{i j}\right|\right) \\
\quad+\frac{1}{2} \sum_{i=1}^{N_{S}} \sum_{j \neq i}^{N_{S}} \phi_{d d}\left(\mathbf{R}_{i j}, \boldsymbol{\mu}_{i}, \boldsymbol{\mu}_{j}\right),
\end{aligned}
$$

where $\phi_{\mathrm{LJ}}(R)$ denotes the usual Lennard-Jones (LJ) interaction for two molecules separated by a distance $R$, and $\phi_{d d}$ is the dipolar interaction energy for two dipoles $\mu_{1}$ and $\mu_{2}$,

$$
\begin{aligned}
& \phi_{d d}\left(\mathbf{R}_{i j}, \boldsymbol{\mu}_{i}, \boldsymbol{\mu}_{j}\right)=-\mu^{2} \frac{D(1,2)}{R^{3}}, \\
& D(1,2)=3\left(\hat{\mu}_{1} \cdot \hat{\mathbf{R}}\right)\left(\hat{\mu}_{2} \cdot \hat{\mathbf{R}}\right)-\hat{\mu}_{1} \cdot \hat{\mu}_{2} .
\end{aligned}
$$

The hats denote unit vectors. Note that $N=N_{S}+N_{\mathrm{LJ}}$ is the total number of molecules in the system.

Henceforth we shall employ reduced units defined in the usual manner. Thus all lengths are measured in units of the Lennard-Jones size parameter $\sigma$ and all energies in units of the Lennard-Jones well depth $\epsilon$. The unit of time is given by $\tau=\sqrt{m \sigma / \epsilon}$ and the dipole moment is given in units of $\mu / \sqrt{\epsilon \sigma^{3}}$.

Molecular dynamics simulations were carried out for mixtures of Lennard-Jones and Stockmayer fluids in which the concentration $x_{s}$ and the dipole moment $\mu$ of Stockmayer molecules was varied. In all simulations, the size parameter $\sigma$ and the energy parameter $\epsilon$ of the Stockmayer and Lennard-Jones system were the same. The simulations were done at a reduced density $\rho=0.822$ and a reduced temperature $T=1.15$. The temperature of the system was held constant with a Nosé heat bath. ${ }^{8}$ Both the pure $L J$ system and the pure Stockmayer system have been studied extensively at this state point. ${ }^{9-11}$

The Nosé equations of motion for a system composed of $N_{L U}$ Lennard-Jones atoms with mass $m$ and $N_{s}$ Stockmayer molecules with mass $m$ and moment of inertia $I$ are: ${ }^{12}$

$$
\begin{aligned}
& m \frac{d^{2} \mathbf{R}_{i}}{d t^{2}}=-\nabla_{R_{i}} V-\frac{1}{s}\left(\frac{d s}{d t}\right)\left(\frac{d \mathbf{R}_{i}}{d t}\right), \quad i=1,2, \ldots, N, \\
& I \frac{d^{2} \boldsymbol{\mu}_{i}}{d t^{2}}=-\nabla_{\mu_{i}} V-\lambda_{i} \boldsymbol{\mu}_{i} \\
&-\frac{1}{s}\left(\frac{d s}{d t}\right)\left(\frac{d \boldsymbol{\mu}_{i}}{d t}\right), \quad i=1,2, \ldots, N_{s}, \\
& Q \frac{d^{2} s}{d t^{2}}=s\left[\sum_{i=1}^{N} m\left(\frac{d \mathbf{R}_{i}}{d t}\right)^{2} \sum_{i=1}^{N} I\left(\frac{d \mu_{i}}{d t}\right)^{2}\right. \\
&\left.\quad-g k_{B} T\right]+\frac{1}{s}\left(\frac{d s}{d t}\right)^{2} .
\end{aligned}
$$

Here $V$ is the total potential energy obtained from Eq. (1), $s$ is the time-scaling parameter introduced by Nosé, and $Q$ is the fictitious mass governing the rate of change of $s$. The parameter $g=\left(3 N_{\mathrm{LJ}}+5 N_{s}-3\right)$ ensures that the instantaneous kinetic energy is balanced by its appropriate value for a system of $N_{\mathrm{LJ}}$ atoms and $N_{s}$ Stockmayer molecules. Finally, $\lambda_{i}$ is a Lagrange multiplier determined from the requirement that $\mu_{i}^{2}=\mu^{2}$ at all times. ${ }^{13}$ A fifth-order predictorcorrector method with a time step $\Delta t=0.0025$ was used for the integration of the equations of motion.

The Ewald sum was used for the evaluation of the dipolar energy, using "tinfoil" boundary conditions. ${ }^{14}$ The convergence parameter $\alpha$ was chosen equal to $6.58 / L$ ( $L$ is the length of the simulation cell) and all reciprocal lattice vectors for which $|\mathbf{k}|^{2} \leqslant 27 / L^{2}$ were included in the reciprocal lattice sum. The real space part was cut off at $L / 2$. Salient details of our simulations are given in Table I. We have tested the number dependence of our results by carrying out simulations at compositions $x_{s}=0.167$ with 108 and 256 particles with $x_{s}=0.164$. The results are compared in Table II. Clearly, the $N$ dependence of the energy and pressure is very small. The small differences observed in the table can be accounted for by the (small) difference in concentration. This was also found to be the case for the dielectric constant. For reasons of economy, most simulations were therefore carried out with 108 particles. The small $N$-dependence was also observed by Pollock and Alder ${ }^{12}$ in their simulations of the pure Stockmayer fluid, and it appears to be a useful characteristic of the Ewald summation.

The free energy of mixing for the system described

TABLE I. Details of molecular dynamics simulations.

\begin{tabular}{lll}
\hline \hline reduced density & $\rho^{*}$ & 0.822 \\
reduced temperature & $T^{*}$ & 1.15 \\
moment of inertia & $I^{*}$ & 0.0025 \\
time-step & $\Delta t$ & 0.0025 \\
No. of time-steps & & 30,000 \\
Nosé mass & $Q^{\mathrm{b}}$ & 100
\end{tabular}

an units of $\left(m \sigma^{2} / \mu^{2}\right)$.

'In units of $\left(m \sigma^{2}\right)^{-1}$. 
TABLE II. Number-dependence of energy $u$, Lennard-Jones energy $u_{L}$, and compressibility factor $Z=B P / \rho$. In these simulations, $\rho=0.822$ and $T=1.15$. Note that the mole fractions are not exactly identical. The small subscripts denote standard deviations.

\begin{tabular}{lrllll}
\hline \hline$N_{S}$ & $N_{\mathrm{LJ}}$ & $\mu^{2}$ & $-u$ & $-u_{\mathrm{LJ}}$ & $Z$ \\
\hline 18 & 90 & 1.5 & $5.489_{5}$ & $5.397_{2}$ & $1.81_{2}$ \\
42 & 214 & 1.5 & $5.492_{2}$ & $5.397_{1}$ & $1.83_{2}$ \\
18 & 90 & 3.0 & $5.798_{5}$ & $5.370_{2}$ & $1.67_{2}$ \\
42 & 214 & 3.0 & $5.797_{4}$ & $5.361_{1}$ & $1.73_{2}$ \\
\hline \hline
\end{tabular}

above can be computed in a particularly simple and elegant manner. Knowledge of the free energy of the pure components is not required. This can be understood by considering a two-stage process in which a fraction $x_{S}$ of Lennard-Jones atoms is changed into Stockmayer molecules with the required dipolar strength $\mu^{2}$.

In the first stage, the Lennard-Jones atoms are changed into molecules with zero dipolar strength and moment of inertia $I$ corresponding to that of the Stockmayer molecules. One thus obtains an ideal mixture of Lennard-Jones and molecular fluids, so that the free energy per particle $a$ (in units of the Lennard-Jones well depth $\epsilon$ ) of the mixture is given by

$$
\begin{aligned}
a_{0}\left(x_{S}\right)= & a_{\mathrm{LJ}}+x_{S} a_{R}^{i d}+T\left[x_{S} \ln x_{S}\right. \\
& \left.+\left(1-x_{S}\right) \ln \left(1-x_{S}\right)\right] .
\end{aligned}
$$

Here $a_{L J}$ is the free energy of the pure Lennard-Jones system and $a_{R}^{i d}$ is the rotational contribution to the free energy. The last term describes ideal mixing.

In the second stage, the Stockmayer molecules are charged to the required value $\mu^{2}$ of the dipolar strength. According to a well-known expression, the free energy change during this process can be obtained as the reversible work required to charge the dipoles of these molecules from a dipolar strength $\mu^{\prime 2}=0$ to the required value $\mu^{12}=\mu^{2}$. Then we may write ${ }^{15}$

$$
a\left(x_{S}\right)-a_{0}\left(x_{S}\right)=\int_{0}^{\mu^{2}}\left\langle\frac{\partial u\left(\mu^{\prime 2}\right)}{\partial \mu^{\prime 2}}\right\rangle_{\mu^{\prime 2}} d \mu^{\prime 2} .
$$

Here $u\left(\mu^{2}\right)$ is the potential energy per particle (in units of $\epsilon$ ) of a system of dipolar strength $\mu^{2}$. The partial derivative of the potential energy with respect to dipolar strength is simply obtained from Eqs. (1)-(3) as

$$
\left\langle\frac{\partial u}{\partial \mu^{2}}\right\rangle=\frac{\left\langle u_{d d}\left(\mu^{2}\right)\right\rangle}{\mu^{2}}
$$

where $u_{d d}$ is the dipolar energy per particle. Hence for the excess free energy per particle, $a$, we obtain

$$
a\left(x_{S}\right)-a_{0}\left(x_{S}\right)=\int_{0}^{\mu^{2}}\left\langle u_{d d}\left(\mu^{\prime 2} ; x_{S}\right)\right\rangle_{\mu^{\prime 2}} \frac{d \mu^{\prime 2}}{\mu^{\prime 2}} .
$$

The free energy of mixing $\Delta a\left(x_{s}\right)$ of the mixture can now be calculated by noting that $x_{S}=1$ corresponds to the pure Stockmayer system. Hence

$$
\begin{aligned}
\Delta a\left(x_{S}\right)= & a\left(x_{S}\right)-\left(1-x_{S}\right) a_{L J}-x_{S} a(1) \\
= & T\left[x_{S} \ln x_{S}+\left(1-x_{S}\right) \ln \left(1-x_{S}\right)\right] \\
& +\int_{0}^{\mu^{2}}\left(\left\langle u_{d d}\left(\mu^{\prime 2} ; x_{S}\right)\right\rangle_{\mu^{\prime 2}}\right. \\
& \left.-x_{S}\left\langle u_{d d}\left(\mu^{\prime 2} ; 1\right)\right\rangle_{\mu^{\prime 2}}\right) \frac{d \mu^{\prime 2}}{\mu^{\prime 2}} .
\end{aligned}
$$

The dipolar energy of the system is readily obtained from the molecular dynamics simulations. This technique has been used previously ${ }^{16}$ to calculate the free energy of the pure Stockmayer system. We note, however, that the free energy of the pure component in not required in Eq. (11).

\section{THERMODYNAMIC PROPERTIES}

In this section, we discuss the results obtained from our simulations and compare these results extensively with the predictions of the perturbation theory proposed by Gubbins and $\mathrm{Twu}^{1,2}$ (GT). The perturbation theory is based on the Padé approximant for the contribution of the dipolar interactions to the Helmholtz free energy, as proposed by Stell $e t$ $a l . .^{3,4}$

$$
a=a_{0}+\frac{a_{2}}{1-\left(a_{3} / a_{2}\right)},
$$

where $a$ is the free energy (per particle) of a reference system and $a_{2}$ and $a_{3}$ are the second- and third-order perturbation terms in the expansion of the free energy in powers of the perturbation, i.e., the dipolar interaction potential, around the reference potential (the first-order term vanishes for a spherical reference potential).

The free energy $a_{0}$ of the (Lennard-Jones) reference mixture is calculated using the van der Waals one fluid conformal solution theory. ${ }^{17-19}$ According to this theory, the pressure $P_{0}$, Helmholtz free energy per particle $a_{0}$, and energy per particle $u_{0}$ of the reference mixture are given as

$$
\begin{aligned}
& P_{0}=P_{x}, \\
& a_{0}=a_{x}+k_{B} T\left[x_{1} \ln x_{1}+x_{2} \ln x_{2}\right], \\
& u_{0}=u_{x},
\end{aligned}
$$

where $P_{0}, a$, and $u_{0}$ are values for a pure fluid with the same state parameters $N, V$, and $T$, and Lennard-Jones interaction parameters $\epsilon$ and $\sigma$ given by

$$
\begin{aligned}
& \sigma_{x}^{3}=\sum_{i} \sum_{j} x_{i} x_{j} \sigma_{i j}^{3}, \\
& \epsilon_{x} \sigma_{x}^{3}=\sum_{i} \sum_{j} x_{i} x_{j} \epsilon_{i j} \sigma_{i j}^{3},
\end{aligned}
$$

where $x_{i}$ is the mole fraction of the $i$ th component in the mixture.

In our case, the reference fluid of course reduces simply to the pure Lennard-Jones liquid characterized by $\epsilon$ and $\sigma$, as the Lennard-Jones parameters of all components in the mixture are identical.

The coefficients $a_{2}$ and $a_{3}$ involve state variables, intermolecular potential parameters and certain integrals $J$ and $K$ over pair and triplet correlation functions of the reference fluid evaluated at reduced temperature $T^{*}=k_{B} T / \epsilon$ and 
reduced density $\rho^{*}=\rho \sigma_{x}^{3}$. Explicit expressions and a table of integrals $J$ and $K$ are given by Gubbins and Twu for a Lennard-Jones reference system. For our simple model, GT predict the ratio $a_{3} / a_{2}$ to be directly proportional to the concentration of polar molecules $x_{s}$.

Various thermodynamic quantities were obtained from our molecular dynamics simulations in the usual manner and collected in Table III. Error estimates (small subscripts) were obtained by computing the standard deviation $s_{N}$ from block averages of successively increasing length as proposed by Flybjerg and Petersen. ${ }^{20}$ From these results, we can construct the excess energy of mixing $u_{e x}\left(x_{S}\right)$ and the free energy of mixing $\Delta a\left(x_{s}\right)$ by integrating the dipolar energy as indicated in the previous section.

In Fig. 1, we display the excess energy as a function of composition for three different values of $\mu^{2}$. The most notable feature here is that $u_{e x}$ is positive and shows an asymmetry with respect to composition, its maximum occurring at compositions rich in nonpolar component. Note also that there is a saturation in $u_{e x}\left(x_{S}\right)$. The predictions of GT are shown in Fig. 1 as well. For low dipole moments, the perturbation theory is seen to underestimate the energy of mixing, while for higher dipole moments the perturbation theory overestimates the excess energy. Also, $u_{e x}\left(x_{S}\right)$ calculated from perturbation theory is rather more symmetric with respect to composition than our simulation results, which show a maximum for compositions rich in nonpolar component. Such a maximum is often observed experimentally in polar/nonpolar fluid mixtures, such as methanol/benzene and ethanol $/ n$-hexane. ${ }^{7}$

The free energy of mixing is most conveniently calculated by fitting the values of the dipolar energy obtained from our simulation to a simple analytic form. We found a simple

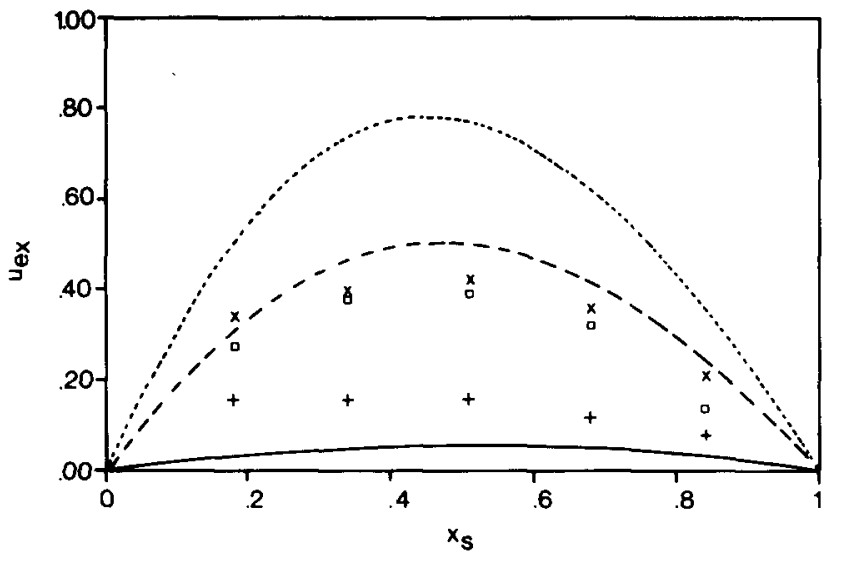

FIG. 1. Excess energy of mixing. The curves are the results of perturbation theory. $-: \mu^{2}=1 ;--: \mu^{2}=3$ and $\cdots:$ The simulation results are marked as points. $\mu^{2}=4,+: \mu^{2}=1 ; \square: \mu^{2}=3$ and $\times: \mu^{2}=4$.

rational form adequate for our purposes:

$$
\left\langle u_{d d}\left(\mu^{2} ; x_{S}\right)\right\rangle=-A x_{s}^{2} \mu^{4} /\left(1+C\left(x_{s}\right) \mu^{2}\right) .
$$

Equation (15) has the correct behavior for small values of $\mu^{2}$, in which case the excess energy over the pure LennardJones system is entirely due to the dipolar energy, whereas for large values of $\mu^{2}$ it follows an upper bound proposed by Onsager $^{21}$ some time ago. The parameter $A$ is simply related to the compressibility factor $Z_{\mathbf{L}}$ and the potential energy $u_{\mathrm{LJ}}$ of the pure $\mathrm{LJ}$ system through ${ }^{22}$

$$
A=\left(T\left(Z_{\mathrm{LJ}}-1\right)-4 u_{\mathrm{LJ}}\right) / 48 T
$$

From our simulations, we find $A=1.70$. In Fig. 2, we show that this behavior is reasonable for the results we obtained. We note that Eq. (15) has the same form as the Padé approx-

TABLE III. Thermodynamic quantities obtained from molecular dynamics simulations. In all simulations, the temperature $T=1.15$ and the density $\rho=0.822$. Also, $u$ denotes the total energy per particle, $u_{d d}$ the dipolar energy per particle, and $Z=\beta P / \rho$ the compressibility factor. Note that all quantities are in reduced units. The

\begin{tabular}{|c|c|c|c|c|c|c|c|c|}
\hline \multirow[b]{2}{*}{$\mu^{2}$} & & \multicolumn{7}{|c|}{ Fraction of Stockmayer particles, $x_{s}$} \\
\hline & & 0.000 & 0.167 & 0.333 & 0.500 & 0.667 & 0.833 & 1.000 \\
\hline & $-u$ & $5.526_{8}$ & $5.518_{3}$ & $5.589_{7}$ & $5.599_{8}$ & $5.658_{9}$ & $5.749_{8}$ & $5.825_{9}$ \\
\hline \multirow[t]{3}{*}{0.50} & $-u_{d d}$ & 0.000 & $0.014_{5}$ & $0.042_{2}$ & $0.096_{2}$ & $0.160_{2}$ & $0.234_{4}$ & $0.316_{3}$ \\
\hline & $Z$ & $2.24 \mathrm{~s}$ & $2.36_{6}$ & $2.11_{4}$ & $2.28_{4}$ & $2.25_{4}$ & $2.11_{3}$ & $2.06_{8}$ \\
\hline & $-u$ & $5.526_{8}$ & $5.585_{5}$ & $5.679_{8}$ & $5.830_{9}$ & 6.026 & $6.219_{8}$ & $6.451_{8}$ \\
\hline \multirow[t]{3}{*}{1.00} & $-u_{d d}$ & 0.000 & $0.033_{5}$ & $0.162_{4}$ & $0.307_{4}$ & $0.500_{4}$ & $0.737_{7}$ & $0.982_{7}$ \\
\hline & $Z$ & $2.24_{5}$ & $2.10_{3}$ & $2.15_{4}$ & $2.00_{4}$ & $1.82_{4}$ & $1.87_{4}$ & $1.71_{4}$ \\
\hline & $-u$ & $5.526_{8}$ & $5.630_{6}$ & $5.854_{8}$ & $6.125_{7}$ & $6.453_{9}$ & $6.822_{9}$ & $7.233_{9}$ \\
\hline \multirow[t]{3}{*}{1.50} & $-u_{d d}$ & 0.000 & $0.104_{3}$ & $0.340_{5}$ & $0.624_{6}$ & $0.974_{6}$ & $1.364_{5}$ & $1.789_{0}$ \\
\hline & $Z$ & $2.24_{5}$ & $2.15_{4}$ & $2.03_{4}$ & $1.84_{3}$ & $1.65_{4}$ & $1.44_{4}$ & $1.24_{4}$ \\
\hline & $-\boldsymbol{u}$ & $5.526_{8}$ & $5.756_{8}$ & $6.188_{10}$ & $6.683_{10}$ & $7.250_{10}$ & $7.866_{11}$ & $8.517_{11}$ \\
\hline \multirow[t]{3}{*}{2.25} & $-u_{d d}$ & 0.000 & $0.227_{5}$ & $0.704_{7}$ & $1.120_{9}$ & $1.837_{8}$ & $2.509_{8}$ & $3.186_{8}$ \\
\hline & $Z$ & $2.24_{5}$ & $2.06_{4}$ & $1.85_{4}$ & $1.53_{4}$ & $1.26_{3}$ & $1.00_{3}$ & $0.52_{4}$ \\
\hline & $-u$ & $5.526_{8}$ & $5.986_{7}$ & $6.606_{10}$ & $7.331_{12}$ & $8.136_{12}$ & $9.049_{11}$ & $9.920_{12}$ \\
\hline \multirow[t]{3}{*}{3.00} & $-u_{d d}$ & 0.000 & 0.497 & $1.174_{12}$ & $1.949_{9}$ & $2.809_{11}$ & 3.793, & $4.726_{12}$ \\
\hline & $Z$ & $2.24_{s}$ & $2.01_{4}$ & $1.72_{4}$ & $1.33_{4}$ & $0.87_{4}$ & $0.42_{4}$ & $-0.10_{4}$ \\
\hline & $-u$ & $5.526_{8}$ & $6.274_{8}$ & $7.302_{10}$ & $8.364_{12}$ & $9.510_{12}$ & $10.724_{12}$ & $12.04_{1}$ \\
\hline \multirow[t]{2}{*}{4.00} & $-u_{d d}$ & 0.000 & $0.827_{\mathrm{g}}$ & $1.949_{21}$ & $3.086_{13}$ & $4.329_{13}$ & $5.648_{12}$ & $7.064_{13}$ \\
\hline & $Z$ & $2.24_{5}$ & $1.91_{4}$ & $1.49_{4}$ & $0.87_{4}$ & $0.27_{4}$ & $-0.28_{4}$ & $-0.98_{3}$ \\
\hline
\end{tabular}
small subscripts denote standard deviations. 


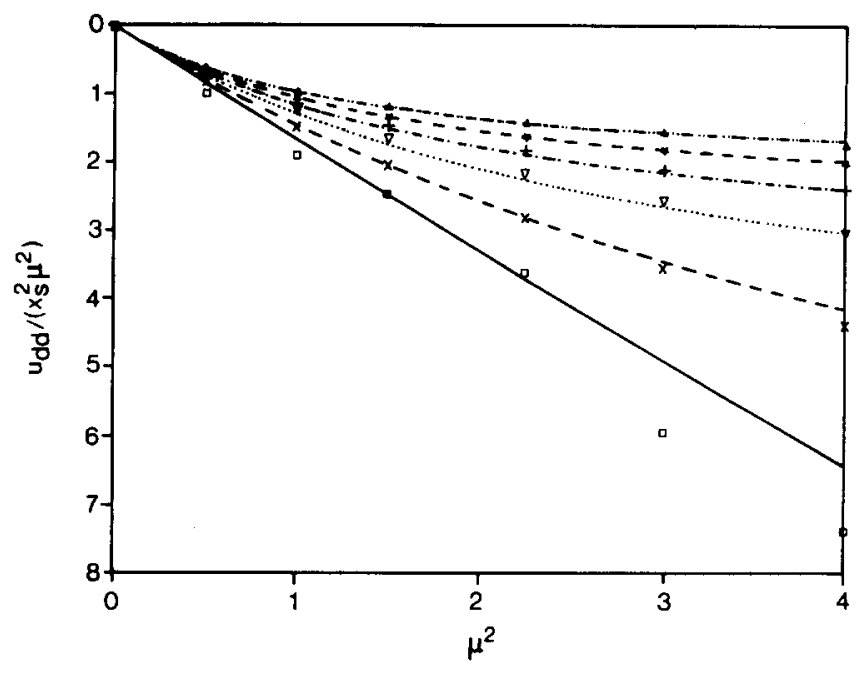

FIG. 2. Dipolar energy $\left\langle u_{d d}\right\rangle /\left(x_{s}^{2} \mu^{2}\right)$ vs $\mu$. The curves give the fit according to Eqs. (15)-(17). - : $x_{s}=0.167 ;--: x_{s}=0.333 ; \cdots: x_{s}=0.500 ; \cdot-\cdot \cdot$ : $x_{s}=0.667 ;--: x_{s}=0.833$ and $-\cdots-: x_{s}=1.000$, The points mark the simulation results. $\square: x_{s}=0.1667 ; \times: x_{s}=0.3333 ; \nabla: x_{s}=0.5000 ;+$ : $x_{s}=0.6667 ; \star: x_{s}=0.8333 ; \Delta: 1.000$ and $\times: \mu^{2}=4$.

imant for the free energy of a dipolar system [Eq. (12)]. In that case, $C\left(x_{s}\right)$ is the ratio $a_{3} / a_{2}$ of the third and second term in the $\lambda$-expansion of the free energy ( $a$ vanishes for the dipolar interaction).

We decided to take $C\left(x_{S}\right)$ as a fitting parameter and found that $C\left(x_{S}\right)$ could be well approximated by

$$
C\left(x_{s}\right)=0.876 x_{s}-0.134
$$

In constructing Eq. (17), we have not used the data for $x_{s}=0.167$, as these are seen to deviate somewhat from the general trend at higher values of $\mu^{2}$. There is evidence that phase separation has occurred for $\mu^{2}=4$, so that these results should not be included in the fit. After substitution of Eqs. (16) and (17) into Eq. (15), an analytic expression for the Helmholtz free energy can be obtained through integration. The final expression differs from Eq. (12) through logarithmic terms, which are generally small. In Fig. 3, we show the excess free energy obtained through this proce-

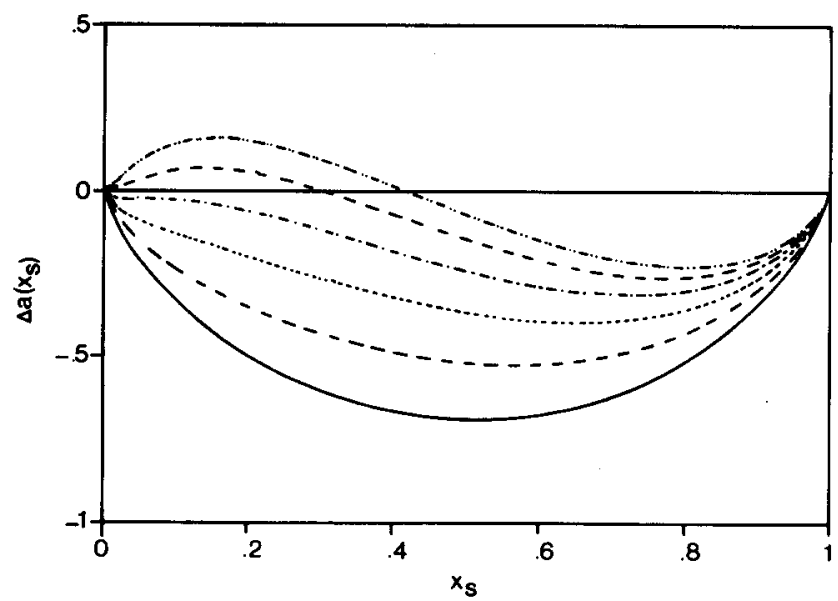

FIG. 3. Excess Helmholtz free energy of mixing for the Stockmayer-Lennard-Jones mixture. $T=1.15$ and $\rho=0.822$. (See also the caption of Fig. 2.)

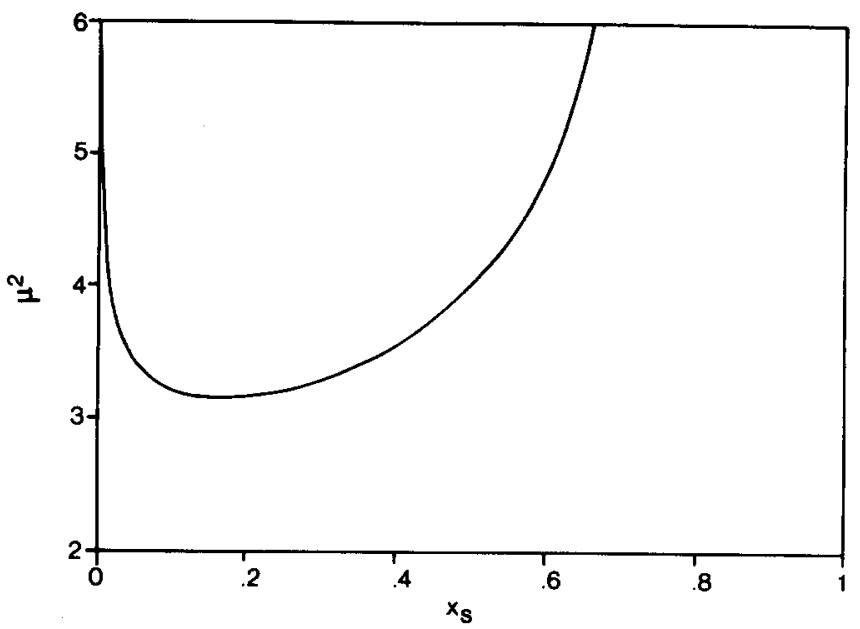

FIG. 4. Phase diagram obtained from the free energy. This according to Eqs. (15) $-(17) . T=1.15$ and $\rho=0.822$.

dure. For larger values of the dipolar strength, it displays a strong asymmetry with respect to composition, with a minimum in regions rich in polar component. As shown before ${ }^{23}$ for values of $\mu^{2}>3.15$, the free energy is no longer a convex function, so that phase separation occurs. In Fig. 4, the composition diagram is shown as obtained from the usual binodal construction. Of course, the phase boundary should be calculated through a binodal construction for the Gibbs free energy of mixing. Our calculation neglects volume effects; we believe these to be small. An estimate of these effects using the perturbation theory of Gubbins and $\mathrm{Twu}^{1,2}$ confirms this assumption. Neglecting volume effects will shift the phase boundaries of the Stockmayer rich phase slightly towards higher concentrations of polar molecules, but the onset of phase separation occurs at almost the same value of $\mu^{2}$. Clearly, a phase separation occurs for $\mu^{2}>3.15$. This result agrees qualitatively with the predictions of perturbation theory, but quantitative differences remain. For exam-

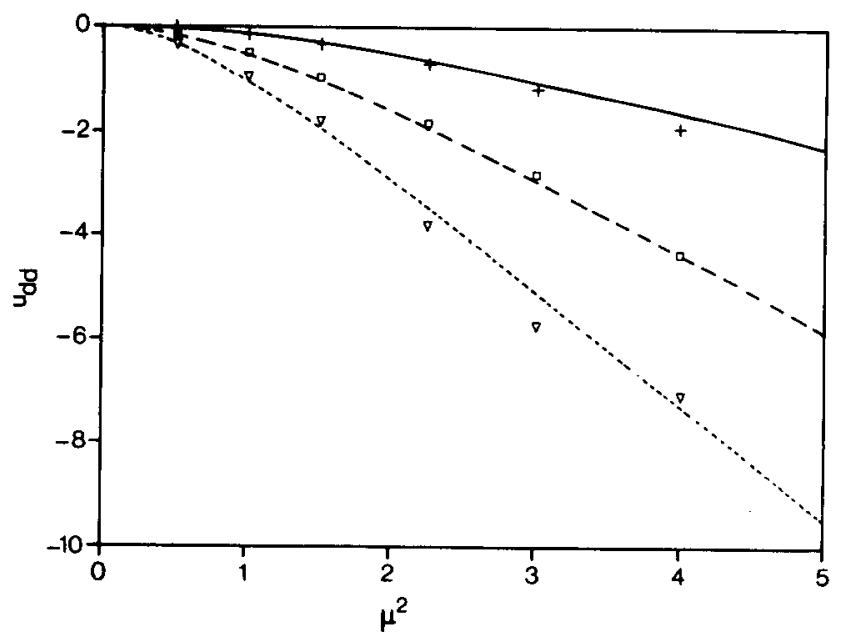

FIG. 5. Comparison of the dipolar energy obtained from simulation with the predictions of perturbation theory. $-: x_{s}=0.333 ;--: x_{s}=0.667$ and $\cdots: x_{s}=1.000$. The simulation results are marked as points. + : $x_{s}=0.333 ; \square: x_{s}=0.667$ and $\nabla: x_{s}=1.000$. 
ple, perturbation theory predicts the onset of phase separation at $\mu^{2} \approx 2.7$.

A more direct comparison with the predictions of perturbation theory can be made by comparing the dipolar energy and the Helmholtz free energy of the mixture with the results of our simulations. According to Eq. (10), the dipolar energy of the fluid can be obtained through differentiation of the Helmholtz free energy with respect to $\mu^{2}$. In Fig. 5 , we compare the dipolar energy obtained through differentiation of Eq. (12) with the results of our simulation. For the pure Stockmayer fluid, the Padé approximant is known to give a fairly accurate representation of the simulation results. ${ }^{3,24,16}$ In the mixture, the Padé approximant for $a\left(x_{s}\right)$ again yields reasonably accurate values for the dipolar energy $\left\langle u_{d d}\right\rangle$. Similarly, the contribution to the free energy from the dipolar interaction obtained from simulation compares very well with the prediction of perturbation theory. Discrepancies appear, however, in the free energy of mixing, as this quantity is obtained as a difference of two relatively large numbers.

Differences between prediction from perturbation theory and the results of our simulation can also be observed in the pressure, as can be seen in Fig. 6 . The overall agreement is fair, but at large dipolar strengths, the theoretical results lie considerably below the results of the simulation, whereas at low values of $\mu^{2}$ these lie above the simulation results. The change in pressure due to the dipolar interactions is considerably less than predicted on the basis of the Padé approximant.

We conclude that perturbation theory gives a fair description of the thermodynamic properties of the mixture, with at least semiquantitative agreement with the results of computer simulations. Differences between theory and simulation do appear in the derivatives of the free energy with respect to density and composition. Moreover, the small differences between GT and simulation results lead to large

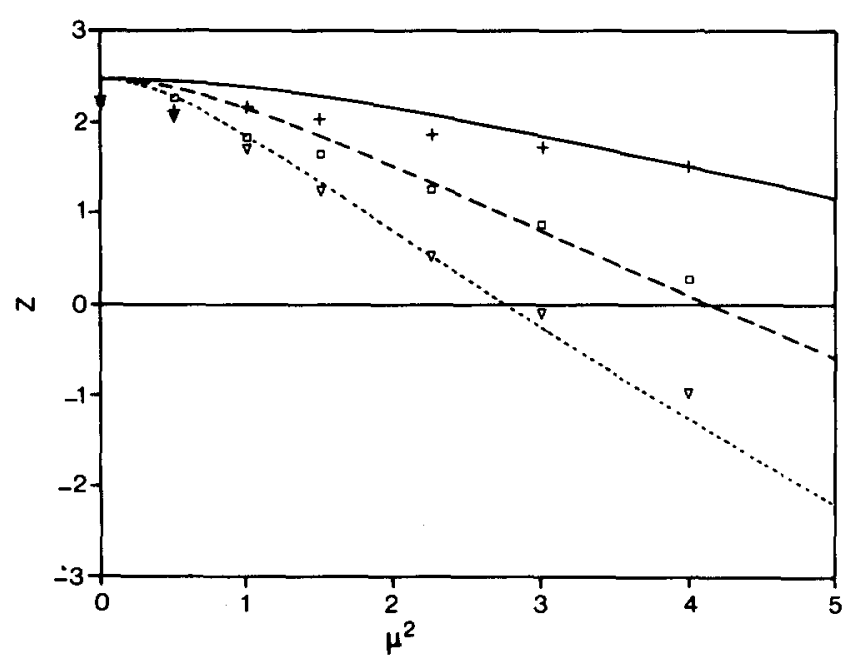

FIG. 6. Compressibility factor $Z=\beta P / \rho$. The points give the results of simulation; the curves the predictions of perturbation theory. $-: x_{s}=0.333$; $: x_{s}=0.667$ and $\cdots: x_{s}=1.000$, $+: x_{s}=0.333 ; \square: x_{s}=0.667$ and $\nabla$ : $x_{s}=1.000$. discrepancies for the excess mixing quantities, such as the excess energy of mixing.

Our simulations confirm the linear dependence of the numerator on the concentration $x_{s}$, although with constants different from theory. This linear dependence is sufficient to give the strong asymmetry of the excess free energy of mixing with respect to composition.

\section{STRUCTURAL PROPERTIES}

The spatial and orientational structural properties of the mixture are most conveniently discussed in terms of the projections of the pair distribution function onto low-order spherical harmonics. For LJ/Stockmayer mixtures, only the distribution function for Stockmayer molecules can be expanded in spherical harmonic coefficients, viz:

$$
\begin{aligned}
g^{s s}(1,2)= & g_{000}^{s s}(R)+h_{D}^{s s} D(1,2) \\
& +h_{\Delta}^{s s}(R) \Delta(1,2)+\cdots,
\end{aligned}
$$

where $g s s(1,2)$ is the pair distribution function for pairs of Stockmayer molecules and $h_{D, \Delta}^{S S}(R)$ are angular correlation functions. Note that $h_{D}^{S S}(R)$ is the projection of $g^{S S}(1,2)$ onto the rotationally invariant angular function $D(1,2)$, defined by Eq. (3). Physically, $h_{D}^{S S}(R)$ is a measure of the mean value of the angular part of the dipolar interaction at separation $R$. It follows that the dipolar energy $\left\langle u_{D D}\right\rangle$ of our system can be expressed in terms of $h_{D}^{S S}(R)$ through

$$
\left\langle u_{d d}\right\rangle=-\frac{2 \pi}{3} \int_{0}^{\infty} \frac{h_{D}^{s s}(R)}{R} d R .
$$

Here $\rho_{s}=N_{s} / V$ is the number density of Stockmayer molecules. Similarly, $h_{\Delta}^{S S}(R)$ is the projection of $g^{S S}(1,2)$ onto $\Delta(1,2)$, defined by

$$
\Delta(1,2)=\hat{\mu}_{1} \cdot \hat{\mu}_{2}=\cos \gamma_{12},
$$

where $\gamma_{12}$ is the angle between the unit vectors $\hat{\mu}_{1}$ and $\hat{\mu}_{2}$. Evidently, $\Delta(1,2)$ is invariant under rotation. Note that $h_{\Delta}^{S S}(R)$ can be viewed as a measure of the alignment of polar molecules separated by a distance $R$.

The distribution function for pairs of unlike molecules $g^{S L}(1,2)$ has the expansion ${ }^{25}$

$$
g^{S L}(1,2)=\sum_{l} g_{l}^{S L}(R) P_{l}\left(\cos \alpha_{12}\right),
$$

where $P_{l}(x)$ is a Legendre polynomal and $\cos \alpha_{12}=\hat{\mu}_{1} \cdot \hat{R}$ is the cosine of the angle between the orientation of the polar molecule $\hat{\mu}_{1}$ and the intermolecular separation $\hat{R}$. Since $g^{S L}(1,2)$ is invariant under change of sign of $\hat{\mu}_{1}$, the expansion (21) contains only even components $l$. In the range of concentrations and dipolar strengths studied here, we may expect the higher angular correlations to be small, particularly since they are caused by three- and higher-body interactions.

We begin by discussing the radial distribution functions ( $r d f$ 's) $g_{000}$. The variation of these functions with dipolar strength depends strongly on composition. Thus for compositions rich in polar component, the distribution function for pairs of Stockmayer particles $g_{000}$ is relatively insensitive to changes in dipolar strength, and its structure is qualitatively similar to that of the pure fluid. Closer examination reveals, 
however, that in contrast to the pure fluid, ${ }^{16}$ its height increases somewhat with $\mu^{2}$. As expected, the peak moves somewhat towards lower values of $R$ for stronger dipole moments. As the fraction of polar molecules decreases, this tendency becomes more pronounced, and at low $x_{s}$, a strong increase of the height of the first peak in $g_{000}$ is observed as $\mu^{2}$ becomes larger, reflecting the increasing tendency of polar molecules to cluster. This can be seen in Fig. 7, where we show $g_{000}$ for three concentrations $\left(x_{s}=0.33,0.50\right.$, and $0.67)$ and various values of $\mu$. In an earlier paper, ${ }^{26}$ we already pointed out the strong asymmetry in local composition with respect to concentration resulting from this behavior. The strong clustering can, of course, be regarded as an onset towards phase separation, which we showed earlier appears to occur at concentrations low in polar component for values of $\mu^{2}>3.15$.

In Fig. 8, we show the rdf's for pairs of $L J$ molecules $g^{L L}$ for two concentrations, $x_{s}=0.33$ and $x_{s}=0.67$. Here, the dependence on concentration is reversed as compared with $g_{000}^{s s}$, the largest changes being observed at high concentrations of Stockmayer molecules. However, the effect is considerably smaller than for Stockmayer molecules. Again this is reflected in the behavior of the local composition numbers, ${ }^{26}$ which do not show the large variation with respect to composition observed for the Stockmayer molecules. The position of the main peak in $g^{L L}$ is seen to be independent of dipolar strength and composition, as expected.

The rdf's for unlike pairs, $g_{0}^{s L}$, are shown in Fig. 9 for the same mole fractions as above. The behavior of these functions appears to be independent of concentration and reflects the increasing dissimilarity of the molecules as the dipolar strength becomes larger.

In Fig. 10, the projection $h_{D}^{S S}(R)$ of the pair distribution function $g^{S S}(1,2)$ onto $D(1,2)$ is shown for various concentrations of polar molecules. The strong increase in angular correlation with decreasing concentration of polar molecules is most notable. Clearly, there is an increasing alignment of polar molecules with decreasing $x_{s}$.

In Fig. 11, we display the projection $h_{\Delta}^{S S}(R)$ for three concentrations and various values of the dipolar strength. We note the same trends as for $h_{D}^{S S}(R)$, namely strong increase in angular correlation as the concentration of Stockmayer molecules decreases, corresponding with an increasing alignment of the polar molecules $[\Delta(1,2)=1]$.

Clearly, both angular correlation functions show a strong increase in orientational ordering as the concentration of polar molecules decreases. This can be understood by considering a highly dilute solution of polar molecules in a nonpolar solvent, so that only pairs of polar molecules are in each others neighborhood. In that case, the molecules are free to align themselves in the energetically most favorable configuration, i.e., head-to-tail for Stockmayer dipoles, for which $D(1,2)=2$. As the concentration of Stockmayer molecules increases, the probability of a third Stockmayer molecule in the vicinity becomes large. It is easily seen that now an optimal configuration cannot be achieved for all pairwise interactions, so that a compromise must be achieved, reducing the alignment of the Stockmayer molecules. This is shown in Fig. 12, where some typical orientations are displayed. For larger concentrations, this "frustration effect" will become increasingly important in determining the angular correlations in our system. This ar-
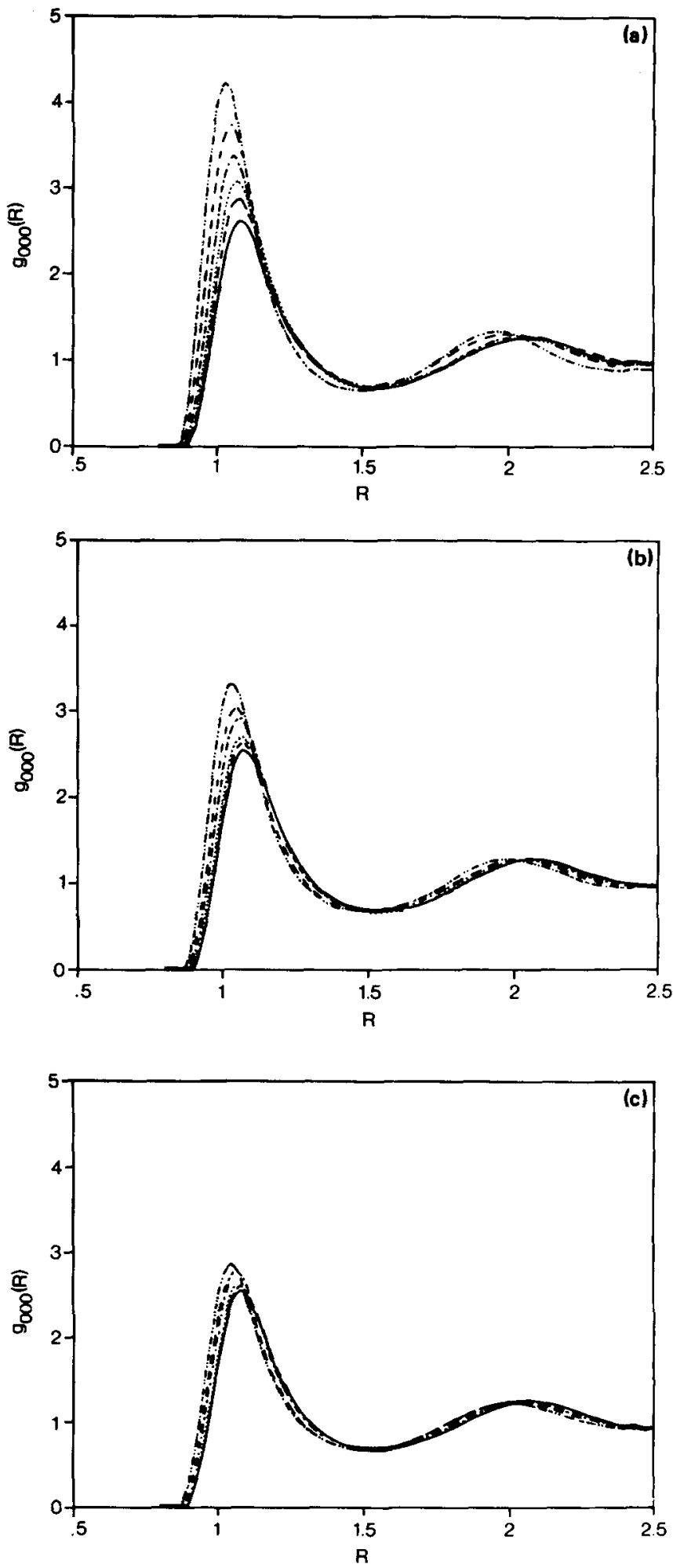

FIG. 7. The radial distribution functions for pairs of Stockmayer molecules at three compositions: (a) $x_{s}=0.33$; (b) $x_{s}=0.50$; (c) $x_{s}=0.67$. $\mu^{2}=0.50 ;--: \mu^{2}=1.00 ; \cdots: \mu^{2}=1.50, \cdot-\cdot \cdot: \mu^{2}=2.25 ;--: \mu^{2}=3.00$ and $\cdots-\cdots: \mu^{2}=4.00$. 

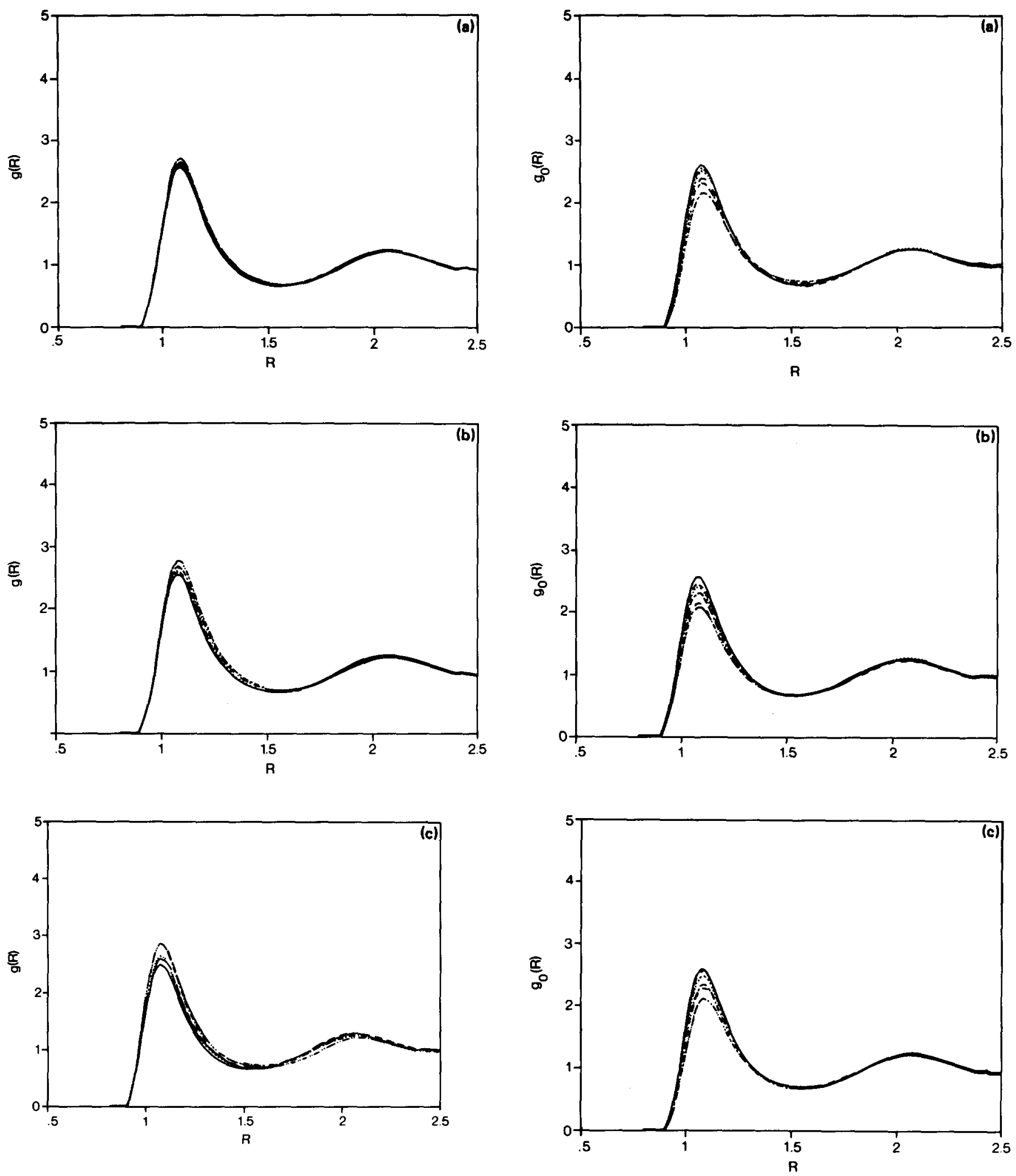

FIG. 8. The radial distribution functions for pairs of Lennard-Jones molecules at three compositions: (a) $x_{s}=0.33$; (b) $x_{s}=0.50$; (c) $x_{s}=0.67$. $\mu^{2}=0.50 ;--: \mu^{2}=1.00 ; \ldots: \mu^{2}=1.50, \cdot-\cdot-\cdot \mu^{2}=2.25 ;--: \mu^{2}=3.00$ and $-\cdots-: \mu^{2}=4.00$.

gument implies an asymmetry in the behavior of the system with respect to concentration, which is reflected in the behavior of the local composition numbers ${ }^{26}$ and the excess properties of mixing.

FIG. 9. The radial distribution functions for pairs of unlike molecules at three compositions: (a) $x_{s}=0.33$; (b) $x_{s}=0.50$; (c) $x_{s}=0.67$. $\mu^{2}=0.50 ;--: \mu^{2}=1.00 ; \ldots: \mu^{2}=1.50, \cdot \cdots \cdot-\cdot: \mu^{2}=2.25 ;--: \mu^{2}=3.00$ and $-\cdots-: \mu^{2}=4.00$.

\section{THE DIELECTRIC CONSTANT}

The angular correlation functions $h_{\Delta}^{S S}(R)$ are a measure of the mean value of $\cos \gamma_{12}$ at a distance $r$ of a polar 

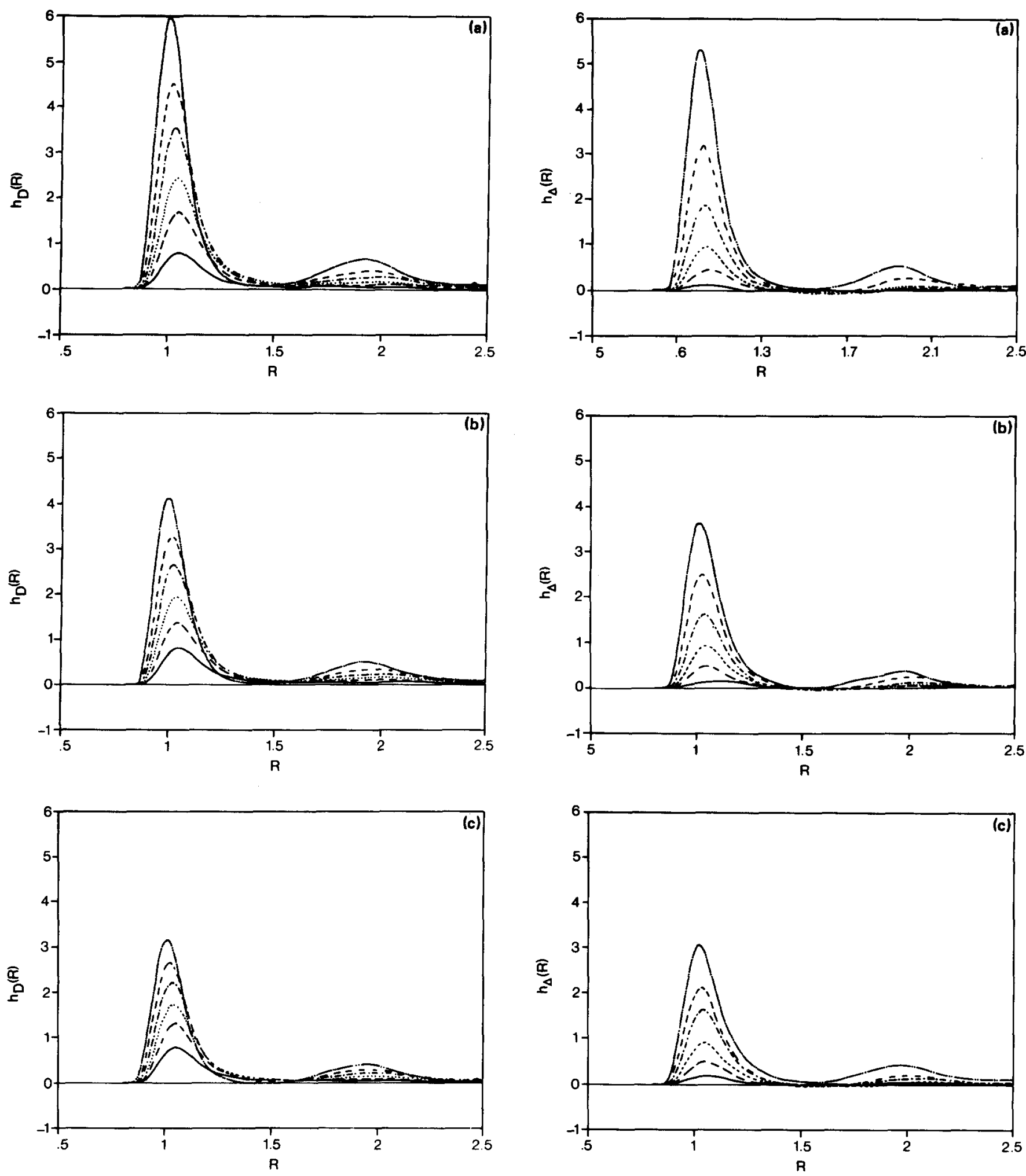

FIG. 10. The angular distribution functions $h_{D}^{s s}(R)$ at three compositions: (a) $x_{s}=0.33$; (b) $x_{s}=0.50 ;$ (c) $x_{s}=0.67$. $-: \mu^{2}=0.50 ;--: \mu^{2}=1.00 ; \ldots$ : $\mu^{2}=1.50, \cdot-\cdot \cdot: \mu^{2}=2.25 ;--: \mu^{2}=3.00$ and $-\cdots-: \mu^{2}=4.00$.

FIG. 11. The angular distribution functions $h_{\Delta}^{\mathrm{ss}}(R)$ at three compositions: (a) $x_{s}=0.33$; (b) $x_{s}=0.50$; (c) $x_{s}=0.67 .-: \mu^{2}=0.50 ;--: \mu^{2}=1.00 ; \ldots$ : $\mu^{2}=1.50, \cdots \cdot-\cdot \mu^{2}=2.25 ;--: \mu^{2}=3.00$ and $-\cdots-: \mu^{2}=4.00$.

molecule. Consequently, the dielectric constant of the system is related to $h_{\Delta}^{S S}(R)$. The Kirkwood correlation factor $g_{k}$ is defined through

$$
g_{k}=1+\frac{4 \pi}{3} \rho \int_{0}^{R_{c}} R^{2} h_{\Delta}^{s s}(R) d R .
$$

The cutoff $R_{c}$ in the integral (22) is necessary because the asymptotic behavior of $h_{\Delta}^{S S}(R)$ depends on the boundary conditions applied to the system, even in the thermodynamic limit. Note that $R_{c}$ is chosen to be large compared to atomic distances, but much smaller than the system size. ${ }^{27}$ With 


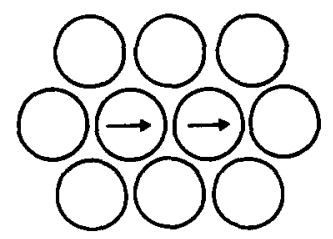

(a)

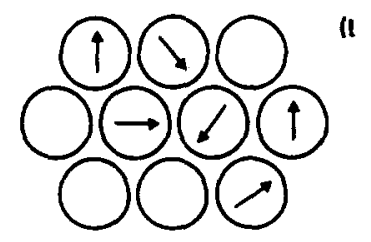

FIG. 12. Illustration of orientational frustration: (a) Nose-to-tail orientations at low concentrations of Stockmayer particles, and (b) orientational frustrations at high concentrations of Stockmayer particles.

this definition, the dielectric constant $\varepsilon$ of our system can be obtained through the Kirkwood formula:

$$
((\varepsilon-1)(2 \varepsilon+1)) / 9 \epsilon=y g_{k},
$$

where $y=4 \pi \beta \rho_{s} \mu^{2} / 9$ is a dimensionless parameter of dipolar strength. In a simulation, the dielectric constant is usually obtained from the fluctuations in dipole moment $\mathbf{M}=\Sigma \boldsymbol{\mu}_{i}$ of the simulation cell. The use of "tinfoil" boundary conditions implies that

$$
\varepsilon=1+3 y g
$$

where the correlation factor $g$ is defined by

$$
g=\left\langle\mathbf{M}^{2}\right\rangle / N_{s} \mu^{2}
$$

In Table IV, the results for the dielectric constant are presented. As expected, $\varepsilon$ increases with dipolar strength and with concentration of polar molecules, with an almost linear dependence on $y$ for small values of $\mu$ and low concentrations $x_{s}$. For larger concentrations and larger values of $\mu$, a much stronger increase is seen.

\section{SUMMARY AND DISCUSSION}

In this paper, we have described the results of molecular dynamics calculations for mixtures of Lennard-Jones and Stockmayer fluids, which can be considered as the simplest model for polar/nonpolar fluid mixtures. The results indicate strongly that the addition of a dipolar interaction between molecules leads to demixing when the polarity of the Stockmayer molecules is large enough $\left(\mu^{2}>3.15\right)$.

The results of our simulation have been compared extensively with the perturbation theory of Gubbins and Twu, ${ }^{1,2}$ based on a Padé approximant ${ }^{3,4}$ for dipolar contribution to the free energy and the van der Waals one fluid conformal solution theory. ${ }^{17-19}$ In general, the perturbation

TABLE IV. Dielectric constant of Stockmayer/Lennard-Jones mixtures. $T=1.15 ; \rho=0.822$.

\begin{tabular}{lllllll}
\hline \hline & \multicolumn{6}{c}{ Fraction of Stockmayer particles, $x_{s}$} \\
\cline { 2 - 7 }$\mu^{2}$ & 0.167 & 0.333 & 0.500 & 0.667 & 0.833 & 1.000 \\
\hline 0.50 & 1.3 & $1.60_{3}$ & $1.90_{5}$ & $2.35_{6}$ & 2.9 & $3.7_{1}$ \\
1.00 & 1.6 & 2.40 & $3.26_{10}$ & $4.36_{15}$ & 5.4 & $7.3_{3}$ \\
1.50 & 1.9 & $3.41_{12}$ & $5.0_{2}$ & $6.7_{4}$ & 10.0 & $13.7_{9}$ \\
2.25 & 2.6 & $4.9_{2}$ & $8.4_{5}$ & $14_{1}$ & 20.2 & $35_{3}$ \\
3.00 & 3.7 & $9.5_{8}$ & $16_{1}$ & $22_{2}$ & 32 & $47_{5}$ \\
4.00 & 6.7 & $16_{2}$ & $23_{3}$ & $50_{7}$ & 74 & $151_{16}$ \\
\hline \hline
\end{tabular}

theory gives a good account of the thermodynamic properties, such as the Helmholtz free energy and the dipolar energy, although slight differences remain. As a result, larger discrepancies appear in derivatives of the free energy, such as the pressure, and the excess mixing properties, the latter being calculated by subtraction of relatively large quantities. The excess energy and free energy of mixing exhibit a strong asymmetry with respect to composition, so often observed in experiment. A linear dependence of the ratio $a_{3} / a_{2}$ of thirdand second-order terms in the perturbation expansion of the free energy, combined with the simple Padé approximant, is sufficient to produce the asymmetric behavior for the excess free energy of mixing.

The radial distribution functions clearly show a tendency of like molecules to cluster when their concentration is low. This effect is much stronger for polar molecules in a nonpolar solvent than vice versa. Moreover, in a nonpolar solvent, the dipolar molecules show a strong tendency to align themselves especially at low concentration of polar molecules. This manifests itself as a strong increase in the height of the main peak in the lower-order spherical harmonic coefficients of the pair distribution function. The decrease of orientational ordering as the concentration of polar molecules increases is thus explained as a frustration effect arising from the impossibility to achieve energetically most favorable orientations when more than two dipoles are in each others vicinity. This frustration effect provides a molecular explanation for the asymmetry observed in the structural and excess mixing properties.

\section{ACKNOWLEDGMENTS}

This research is part of the research program of the foundation for Chemical Research of the Netherlands (SON), financially supported by the Netherlands Organization of Scientific Research.

${ }^{1}$ K. E. Gubbins and C. H. Twu, Chem. Eng. Sci. 33, 863 (1977).

${ }^{2}$ K. E. Gubbins and C. H. Twu, Chem. Eng. Sci. 33, 879 (1977).

${ }^{3}$ G. Stell, J. C. Rasaiah, and H. Narang, Mol. Phys. 23, 393 (1972).

${ }^{4}$ G. Stell, J. C. Rasaiah, and H. Narang, Mol. Phys. 27, 1393 (1974).

${ }^{5}$ M. Wojcik and K. E. Gubbins, Mol. Phys. 51, 951 (1984).

${ }^{6} \mathrm{G}$. Morriss and D. Isbister, Mol. Phys. 51, 911 (1986).

${ }^{7}$ J. S. Rowlinson, Fluids and Fluid Mixtures (Butterworth Scientific Publications, London, 1959).

${ }^{8}$ S. Nosé, J. Chem. Phys. 81, 511 (1984).

${ }^{9}$ M. Neumann, O. Steinhauser, and G. S. Pawley, Mol. Phys. 52, 97 (1984).

${ }^{10}$ D. J. Adams and E. M. Adams, Mol. Phys. 42, 907 (1981).

${ }^{11}$ M. Neumann, Mol. Phys. 50, 841 (1983).

${ }^{12}$ E. L. Pollock and B. J. Alder, Physica A 102, 1 (1980).

${ }^{13}$ E. Edberg, D. Evans, and G. P. Morris, J. Chem. Phys. 84, 6933 (1986).

${ }^{14}$ S. W. de Leeuw, J. W. Perram, and E. R. Smith, Ann. Rev. Phys. Chem. 37, 240 (1986).

${ }^{15}$ D. Chandler, $A$ Modern Introduction to Statistical Mechanics (Oxford U.P., New York, 1987).

${ }^{16}$ L. Verlet and J. J. Weis, Mol. Phys. 28, 665 (1974).

${ }^{17}$ T. W. Leland, J. R. Rowlinson, and G. A. Sathers, Trans. Faraday Soc. 68, 1447 (1968).

${ }^{18}$ C. H. Twu, K. E. Gubbins, and C. G. Gray, Mol. Phys. 29, 713 (1975).

${ }^{19}$ I. R. McDonald, "Statistical mechanics," in Specialist Periodical Report, edited by K. Singer (The Chemical Society, 1973).

${ }^{20}$ H. Flyberg and H. G. Petersen, J. Chem. Phys. 91, 461 (1989). 
${ }^{21}$ L. Onsager, J. Phys. Chem. 43, 189 (1939).

${ }^{22}$ I. R. McDonald, J. Phys. C 7, 1225 (1974).

${ }^{23}$ S. W. de Leeuw, C. P. Williams, and B. Smit, Mol. Phys. 65, 1269 (1988)

${ }^{24}$ B. Smit, C. P. Williams, E. M. Hendriks, and S. W. de Leeuw, Mol. Phys. 68, 765 (1989).
${ }^{25}$ C. G. Gray and K. E. Gubbins, Theory of Molecular Fluids. I. Fundamen tals (Clarendon, Oxford, 1984).

${ }^{26}$ S. W. de Leeuw, C. P. Williams, and B. Smit, Fluid Phase Equilibria 48 99 (1989).

${ }^{27}$ J. G. Kirkwood, J. Chem. Phys. 7, 911 (1939). 